\title{
AVALIAÇÃO DE MÉTODOS QUÍMICOS PARA PREDIZER A DISPONIBILIDADE DO NITROGÊNIO ORGÂNICO NO SOLO ${ }^{(1)}$
}

FRANCISCO SOLANO DE OLIVEIRA RODRIGUES FILHO (2), Seção de Oleaginosas, Instituto Agronómico, e JAMES D. LANCASTER (3), Universidade do Estado do Mississipi (EUA).

\section{RESUMO}

Vários métodos químicos recomendados para predizer a disponibilidade do nitrogênio orgânico no solo foram avaliados, usando-se nitrogênio mineralizável pela incubação aeróbica e araeróbica, em condiçōes de laboratório, como padrōes. Sessenta e duas amostras de solo, variando amplamente em pH, textura, matéria orgânica e manejo da gleba, foram coletadas de áreas representativas do Estado do Mississippi. Baseados no grau de consistência de correlação com o nittrogênio mineralizado por ambas as incubaçōes, aeróbica e anaeróbica, os métodos químicos se classificaram na seguinte ordem, como indices de disponibilidade do nitrogênio orgânico no solo, usando-se análise de correlação simpiples: hídrôlıses com $\mathrm{Ca}(\mathrm{OH}),>$ nitrogênio total $>$ digestão com permanganato alcalino $>$ nitrogênio pelo método Walkley-Black $>$ métodos de autoclavagens > matéria orgânica > absorção pela luz ultravioleta. Essa ordem foi inalterada pela inclusão da relação carbono:nitrogênio (C:N) aos métodos biológicos, usando-se regressão múltipla e análises de correlação, exceto no mẻtodo da matẻria orgânica, que passou então a situar-se entre os métodos quimicos, apresentando as maiores correlaçōes com os biologicos.

(1) Este trabalho faz parte da Tese para obtençăo do título de Ph.D. do primeiro autor pela Universidade do Estado do Mississipi (EUA), em julho de 1981. Recebido para publicacão a 2 de dezembro de 1981.

(2) Com bolsa de suplementação do CNPq.

(3) Professor de Agronomia da Universidade do Estado do Mississipi (EUA). 


\section{INTRODUGÃO}

Métodos biológicos e químicos têm sido utilizados para predizer a disponibilidade do nitrogênio orgânico no solo, conforme boa revisão feita por BREMNER (3). Os métodos biológicos têm como princípio a produção de nitrogênio mineral durante incubação (aeróbica ou anaeróbica), sob condiçôes controladas de laboratório ou sob condiçōes naturais de campo, segundo FITTS et alii (5), STANFORD \& HANWAY (22), MUNSON \& STANFORD (11), MacLEAN (10), STANFORD et alii (20), STANFORD \& SMITH (23), SMITH et alii (18) e POWERS (12).

Os métodos biológicos, considerados mais satisfatórios do que os químicos para predizer a disponibilidade do nitrogênio orgânico às plantas, têm uma substancial desvantagem para uso em análises de rotina, porque os procedimentos de incubação são demorados e tediosos para serem executados.

Nos métodos químicos, com exclusão daqueles da małéria orgânica do nitrogênio total e da absorção pela luz ultravioleta, o princípio envolvido é a extração de várias formas de nitrogênio orgânico em proporção às subseqüentes quantidades disponiveis às plantas, por meio da mineralização, segundo CORNFLELD (4), STANFORD \& DEMAR (21), FOX \& PIEKIELEK (6) e BARYOSEF \& AKIRI (1).

De acordo com KEENEY \& BREMNER (8), os métodos químicos para avaliação da disponibilidade do nitrogênio no solo são de grande importância: geralmente mais precisos e mais rápidos do que os biológicos, não são tão afetados pelo armazenamento das amostras de solo antes das análises. Entretanto, os biológicos são de grande ajuda como padrões para a avaliação dos métodos químicos: muitos destes têm sido propostos como índices da disponibilidade do nitrogênio crgânico e comparados com os biológicos mediante técnicas de regressão e análises de correlação, segundo BREMNER (3). O objetivo principal deste estudo foi reavaliar alguns métodos químicos propostos anteriormente para predizer a disponibilidade do nitrogênio orgânico e mais dois propostos por STANFORD (1), usando-se como padrōes para comparação a mineralização aeróbica e anaeróbica do nitrogênio orgâniç, sob condiçōes controladas de incubação.

\section{MATERIAL E METTODOS}

Para o estudo, foram coletadas cinqüenta e nove amostras de solos de áreas agricultáveis e não agricultáveis do Estado de Mississipi (EUA)

(4) STANFORD, G. U.S. Soils Laboratory U.S.D.A., Beltsville, Maryland. Comunicação pessoal. 
a uma profundidade de $0-15 \mathrm{~cm}$ e três amostras a uma profundidade de $15-30 \mathrm{~cm}$.

As áreas de amostragem de solos foram escolhidas de maneira a se obter uma ampla variação no conteúdo de matéria orgânica, textura do solo e práticas agrícolas.

As amostras de solos foram secas sem demora, espalhadas sobre um banco na estufa e passadas em peneira de 10 "mesh". As amostras para análises foram colocadas em sacos plásticos armazenados em caixas de papelão parafinadas.

\section{Métodos usados para predizer a disponibilidade do nitrogênio no solo}

Os seguintes métodos foram utilizados para predizer a disponibilidade do nitrogênio orgânico no solo:

1. Matéria orgânica, pelo método da Universidade de Auburn PROCEDURES ... (14) - M1.

2. Nitrogênio total, pelo método modificado proposto por BREMNER (2) - M2.

3. Amônio trocável e nitrogênio inorgânico inicial, pelo método de SAHRAWAT \& PONNAMPERUMA (16) - M5 e M4.

4. Hidrólises pelo hidróxido de cálcio, pelo método proposto por PRASAD (13) - M3.

5. Autoclavagem com cloreto de cálcio $0,01 \mathrm{M}$ e modificações, pelo método de STANFORD (19) - M6, Mr e M8.

6. Absorção pela luz ultravioleta, pelo método proposto por FOX \& PIEKIELEK (6) - M15.

7. Mineralização aeróbica do nitrogênio orgânico do solo, pelo método de STANFORD et alii (20) — M9, M10 e M12.

8. Mineralização anaeróbica do nitrogênio orgânico do solo, pelo método proposto por SNITH \& STANFORD (17) — M11.

Além desses, foram testados outros dois como sugestão pessoal do Dr. G. Stanford, com algumas modificaçōes: M13 e M14.

9. Determinação do nitrogênio pela oxidação com dicromato de potássio (Walkley-Black-N) - M13. Nessa sugestão do Dr. G. Stanford, o princípio de oxidação é o mesmo empregado na determinação da matéria orgânica pelo método de Walkley-Black:

" $1 \mathrm{~g}$ de solo seco ao ar, passado em peneira 40 mesh, foi colocado em um frasco de $250 \mathrm{ml}$, ao qual foram adicionados exatamente $15 \mathrm{ml}$ de $\mathrm{K}_{2} \mathrm{Cr}_{2} \mathrm{O}_{7} 1 \mathrm{~N}$ e $30 \mathrm{ml}$ de $\mathrm{H}_{2} \mathrm{SO}_{4}$ concentrado. Ao conteúdo, agitado por um minuto e deixado descansar por 30 minutos, foram adicionados $100 \mathrm{ml}$ 
de água destilada, dando um volume final de aproximadamente $125 \mathrm{ml}$. Uma alíquota de $100 \mathrm{ml}$ foi transferida para um frasco Kjeldahl, adicionando-se-lhe $200 \mathrm{ml}$ de água destilada, algumas bolinhas de vidro e $30 \mathrm{ml}$ de $\mathrm{NaOH} 40 \%$. Nitrogênio amoniacal foi separado por destilação e o destilado, coletado por 30 minutos em uma solução pađrão de ácido sulfúrico. O destilado foi completado a um volume específico e, a concentração de amônio, determinada colorimetricamente, usando-se reagentes de Nesler, de acordo com LANCASTER (9)".

10. Determinação do nitrogênio pela digestão com permanganato de potássio alcalino - M14:

" $1 \mathrm{~g}$ de solo seco ao ar, passado em peneira de 40 mesh, foi transferido para um frasco Kjeldahl de $500 \mathrm{ml}$ com $2 \mathrm{~g}$ de $\mathrm{KMnO}_{4}, 300 \mathrm{ml}$ de água destilada e $1 \mathrm{~g}$ de $\mathrm{MgO}$ em pó. $\mathrm{O}$ frasco foi ligado a um aparelho destilador e, o destilado, coletado por 30 minutos em $50 \mathrm{ml}$ de ácido bórico $4 \%$ contendo cinco gotas do indicador verde de bromo cresol-vermelho de metila. O amônio foi titulado, usando-se uma solução padrão de $\mathrm{H}_{2} \mathrm{SO}_{4} . "$

\section{Cálculo das relações carbono:nitrogênio}

As relações carbono:nitrogênio foram calculadas usando-se o conteúdo de carbono $(58 \%)$ da matéria orgânica e, o nitrogênic, pelo método Kjeldahl (nitrogênio total) - M16 - e Walkley-Black (digestão com $\mathrm{K} . \mathrm{Cr}_{2} \mathrm{O}_{7}$ e $\left.\mathrm{H}_{2} \mathrm{SO}_{4}\right)$ - M17.

\section{Análises estatisticas}

Análise de regressão foi utilizada na avaliação dos resultados, pelo uso do "Mafes Regression Program", provido pelo Devartamento de Estatística Experimental da Universidade do Estado do iNississipi (EUA).

As quantidades de nitrogênio orgânico mineralizado pelas incubações aeróbicas e anaeróbicas (métodos biológicos) foram correlacionadas com as de nitrogênio determinado pelos métodos quimicos, usando-se análise de regressão simples. Quando as relaçōes $C: N$ furam incluídas nos estudos de correlação, adotaram-se análises de regressão múltipla. Nesse caso, os métodos biológicos foram sempre tomados como variáveis dependentes e, os químicos e as relações $\mathrm{C}: \mathrm{N}$, como variáveis independentes. Os resultados obtidos no estudo, sobre relações com a classe textural dos solos, não são apresentados neste trabalho.

Os vários métodos de análises e suas respectivas abreviaturas são mostrados no quadro 1. 
QUADRO 1. Designação abreviada dos métodos e fatores do solo usados para predizer a disponibilidade do nitrogênio orgânico do solo

Método ou fator do solo

Designação abreviada

Matéria orgânica M1

Nitrogênio total M2

Hidrólises com $\mathrm{Ca}(\mathrm{OH})$. M3

Nitrogênio inorgânico M4

Amònio trocável M5

Autoclavagem com $\mathrm{KCI} 1 \mathrm{M}$ M6

Autoclavagem com $\mathrm{CaCl}_{2}, 0,01 \mathrm{M}$ M7

Autoclavagem com $\mathrm{CaCl}_{2}, 0,01 \mathbf{M}-\mathrm{KCl} \mathbf{1 M}$ M8

Mineralização aeróbica - uma semana M9

Mineralização aeróbica - duas semanas M10

Mineralização anaeróbica - três semanas M11

Mineralização aeróbica - uma semana - duas semanas M12

Nitrogênio pelo Walkley-Black M13

M14

M15

M16

Relação $\mathrm{C}: \mathrm{N}$ (Nitrogênio pelo Kjeldahl

M17

\section{RESULTADOS E DISCUSSÃO}

As médias de três determinações e variaçōes das quantidades de nitrogênio obtidas pelos métodos usados, são apresentadas no quadro 2 . Os conteúdos de matéria orgânica e nitrogênio total dos solos são dados em partes por milhão (ppm) somente por conveniência $\epsilon$ uniformidade na apresentação dos resultados. Estes podem ser divididos por 10.000 para convertê-los em percentagem, o que é o método mais comum de representação de matéria orgânica e nitrogênio total.

O quadro 3 mostra os coeficientes de correlação simples ( $r$ ) entre os métodos químicos. Como era esperado, nitrogêrio inorgânico inicial, M4, e amônio trocável, M5, foram fracamente correlacionados com os outros métodos químicos, como também a absorção ultravioleta, M15, apesar de acusar valores mais altos do coeficiente de correlação. A absorção pela luz ultravioleta, M15, correlacionou melhor com matéria orgânica, M1, e com os métodos de autoclavagens (M6, M7 e M8) do que com os outros métodos químicos. O coeficiente de correlação entre os outros métodos químicos variou de 0,624 a 0,929 , sendo o maior valor observado entre nitrogênio total, M2, e hidrólises com $\mathrm{Ca}(\mathrm{OH})_{2}, \mathbf{M 3}$. 
QUADRO 2. Média e variação nas quantidades de nitrogênio determinadas pelos diferentes métodos usados para predizer a disponibilidade do nitrogênio orgânico do solo

\begin{tabular}{|c|c|c|c|c|}
\hline \multirow{2}{*}{$\begin{array}{c}\text { Método (1) } \\
\text { M1 (2) }\end{array}$} & \multirow{2}{*}{ Média } & \multicolumn{3}{|c|}{ Variação } \\
\hline & & $54.800,0$ & - & $4.200,0$ \\
\hline M2 & 860,0 & $2.500,0$ & - & 270,0 \\
\hline M3 & 71,4 & 201,1 & - & 30,0 \\
\hline M4 & 20,4 & 53,7 & - & 7,4 \\
\hline M5 & 8,7 & 16,6 & - & 2,7 \\
\hline M6 & 67,8 & 195,2 & - & 17,9 \\
\hline M7 & 37,6 & 118,2 & - & 4,0 \\
\hline M8 & 43,8 & 158,5 & - & 11,0 \\
\hline M9 & 14,6 & 59,9 & - & 2,9 \\
\hline M10 & 15,0 & 39,4 & - & 4,6 \\
\hline M11 & 35,7 & 144,1 & - & 2,0 \\
\hline M12 & 29,6 & 86,6 & - & 7,0 \\
\hline M13 & 553,4 & $2.004,0$ & - & 176,0 \\
\hline M14 & 145,1 & 498,5 & - & 26,6 \\
\hline M15 & 0,32 & 1,1 & - & 0,17 \\
\hline M16 (4) & 9,7 & 20,3 & - & 4,1 \\
\hline M17 (4) & 10,4 & 24,5 & - & 4,3 \\
\hline
\end{tabular}

(1) Ver abreviação dos métodos no quadro 1. (2) Conteúdo de matéria orgânica.

(3) Absorbancia a $260 \mathrm{~nm}$. (4) Relação C:N.

PRASAD (13) também achou alta correlação, $r=0,771$, entre nitrogênio total e o determinado pelo $\mathrm{Ca}(\mathrm{OH})_{2}$. Como era de esperar, houve alta correlação entre nitrogênio total, M2, e matéria orgânica, M1, sendo o valor $\mathrm{r}=0,879$. As quantidades de nitrogênio obtidas pela hidrólise com $\mathrm{Ca}(\mathrm{OH})_{2}$, nitrogênio pelo Walkley-Black, M13, e digestão com permanganato alcalino, M14, em quase todos os casos, foram mais altamente correlacionadas com nitrogênio total, M2, e matéria orgânica, M1, do que as determinadas pelos procedimentos de autoclavagem (M6, M7, M8).

Com base nos baixos coeficientes de correlação, parece ser altamente provável que os potenciais dos métodos M4, M5 e M15, para predizer a disponibilidade do nitrogênio orgânico do solo, sejam diferentes daqueles dos outros métodos químicos e, provavelmente, não muito altos. 


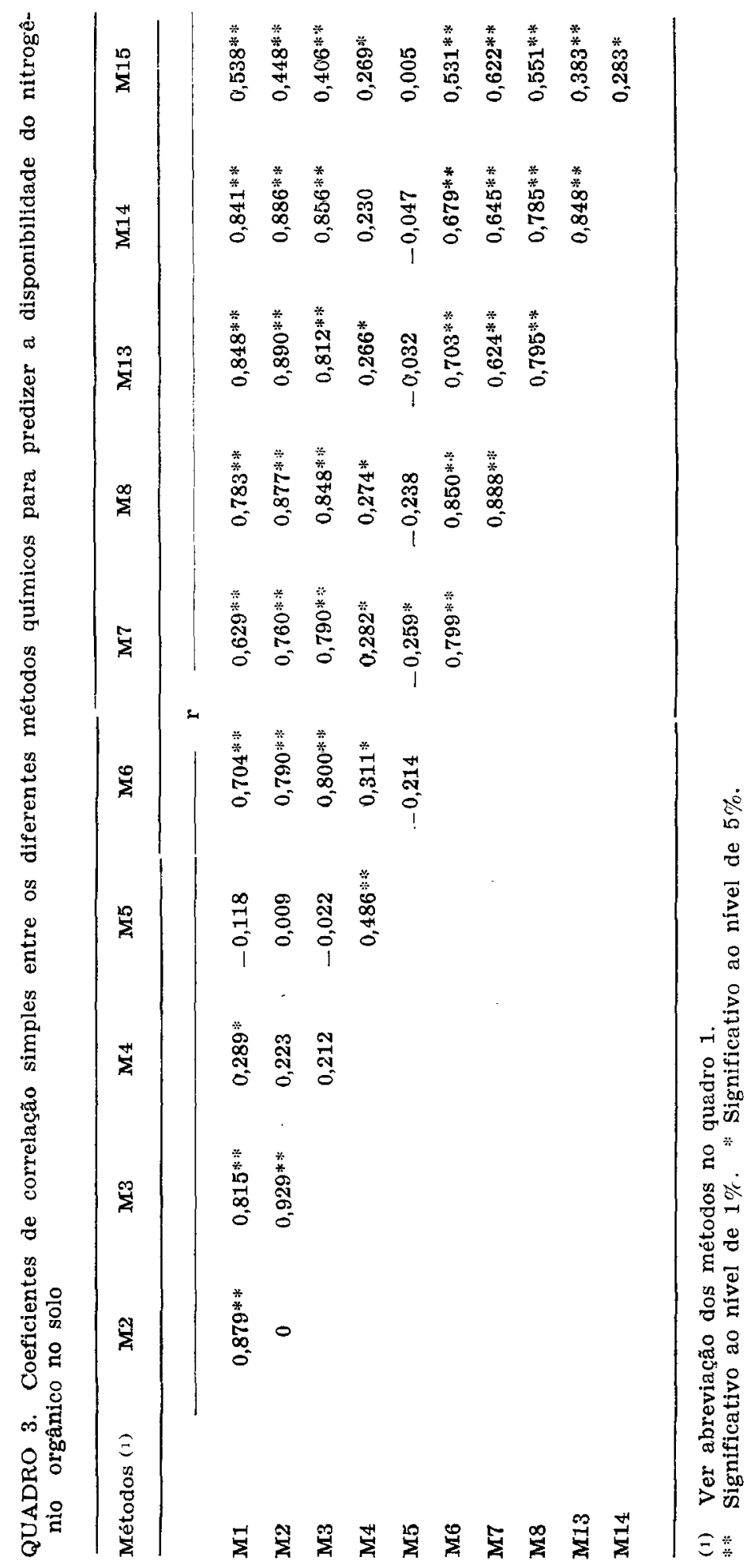


Os coeficientes de determinação $\left(r^{2}\right)$ nas correlaçōes entre o nitrogênio mineralizado sob condições aeróbicas e anaeróbicas e as quantidades de nitrogênio determinadas pelos métodos químicos são mostrados no quadro 4.

QUADRO 4. Coeficientes de determinação $(r 2)$ nas correlações entre o nitrogênio mineralizado sob condições aeróbica e anaeróbica e as quantidades de nitrogênio obtidas pelos métodos químicos

\begin{tabular}{|c|c|c|c|c|}
\hline \multirow{2}{*}{ Métodos (1) } & \multicolumn{3}{|c|}{ Aeróbica } & \multirow{2}{*}{ 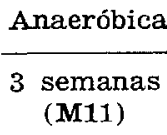 } \\
\hline & $\begin{array}{c}1 \text { semana } \\
\text { (M9) }\end{array}$ & $\begin{array}{l}2 \text { semanas } \\
\text { (M10) }\end{array}$ & $\begin{array}{c}1+2 \text { semanas } \\
(\text { (M12) }\end{array}$ & \\
\hline M1 (2) & $0,555 * *$ & $0,362 * *$ & $0,534 * *$ & $0,440 \% *$ \\
\hline $\mathbf{M} 2$ & $0,726 * *$ & $0,540 * *$ & $0,736 * *$ & $0,686^{* *}$ \\
\hline M3 & $0,746^{* *}$ & $0,634 * *$ & $0,796 *$ & $0,667^{* *:}$ \\
\hline M4 & 0,031 & 0,048 & 0,043 & 0,055 \\
\hline M5 & 0,003 & 0,004 & 0,002 & 0,006 \\
\hline M6 & 0,666 & $0,414 *$ & $0,631 *$ & $0,512 *$ \\
\hline $\mathbf{M 7}$ & $0,670 * *$ & 0,477 & $0,666 * *$ & $0,565^{* *}$ \\
\hline M8 & $0,726 *$ & $0,430 * *$ & $0,675 *$ & 0,530 \\
\hline M13 & $0,602 * \%$ & $0,437 *$ & $0,604 * *$ & $0,559 *$ \\
\hline M14 & $0,602 * *$ & $0,520 * *$ & $0,635 \%$ & $0,602^{* *}$ \\
\hline M15 & 0,238 & $0, \mathbf{0} 88$ & 0,189 & 0,142 \\
\hline
\end{tabular}

(1) Ver abreviação dos métodos no quadro 1 . (2) Conteúdo de matéria orgânica.

(3) Absorbância pela luz ultravioleta a $260 \mathrm{~nm}$.

** Significativo ao nível de $1 \%$.

Esses resultados mostram que, com exceção do nitrogènio inorgânico inicial (M4), do amônio trocável (M5) e da absorção pela luz ultravioleta (M15), todos os métodos químicos foram altamente correlacionados com os biológicos. Mineralização aeróbica pelo M10 (curto intervalo da técnica de incubação proposta por STANFORD et alii (20), para uso do cálculo do nitrogênio potencialmente mineralizado) correlacionou-se menos com os métodos químicos do que os outros métodos biológicos. O nitrogênio total (M2) e hidrólise com $\mathrm{Ca}(\mathrm{OH})_{2}$ (M3) deram os valores mais altos e mais consistentes de correlação com os métodos biológicos, seguindo-se a digestão com permanganato alcalino (M14), os procedimentos de autoclavagem (M6, M7, M8), nitrogênio pelo Walkley-Black (M13), matéria orgânica (M1) e, finalmente, absorção pela luz ultravioleta (M15).

O procedimento de autoclavagem, M7, proposto em 1969 por STANFORD \& DEMAR (21) não foi altamente correlacionado com a mineralização aeróbica, M10, nem com a anaeróbica, M11, como mencionado por SMITH \& STANFORD (17). 
Os resultados obtidos nesta investigação, com a absorção pela luz ultravioleta, M15, não concordam com os obtidos por FOX \& PIEKIELEK (6), indicando ser este método bastante eficiente para predizer a disponibilidade do nitrogênio orgânico do solo.

A fỉm de investigar a influência da relação carbono:nitrogênio $(\mathrm{C}: \mathrm{N})$ sobre a relação entre os métodos biológicos e químicos, foi realizada uma análise de regressão múltipla: os biológicos foram tratados como variáveis dependentes e, os químicos e as relações $C: N$, como variáveis independentes.

Coeficientes de determinação $\left(\mathrm{R}^{2}\right)$ para todas as combinações possíveis com os métodos biológicos são mostradas nos quadros 5 e 6 . Pode-se observar que todos os métodos químicos, com exceção de nitrogênio inorgânico inicial (M4), do amônio trocável (M5) e da absorção pela luz ultravioleta (M15), foram altamente correlacionados com os métodos biológicos mais as relações carbono:nitrogênio. A inclusão das relaçôes $\mathrm{C}: \mathrm{N}$ na análise de regressão aumentou substancialmente os valores de $\mathrm{R}^{2}$ somente para matéria orgânica (M1) : isso pode ser cioservado, comparando-se os valores de $R^{2}$ do quadro 4 com os valores de $R^{2}$ dos quadros 5 e 6. A relação $C: N$ baseada no nitrogênio Kjeldahl (M16) e a baseada no nitrogênio Walkley-Black (M17) proporcionaram resultados similares de $\mathrm{R}^{2}$ na regressão múltipla e análises de correlação.

QUADRO 5. Coeficientes de determinação ( $R$ 2) nas correlações entre nitrogênio determinado pelos vários métodos químicos e aquele mineralizado pela incubação aeróbica e anaeróbica e a relação $\mathrm{C}: \mathrm{N}$ ( $\mathrm{N}$ do Kjeldahl)

\begin{tabular}{|c|c|c|c|c|}
\hline \multirow{3}{*}{ Métodos (1) } & \multicolumn{4}{|c|}{ M16 } \\
\hline & \multicolumn{3}{|c|}{ Aeróbica } & \multirow{2}{*}{$\frac{\text { Anaeróbica }}{3 \text { semanas }}$} \\
\hline & 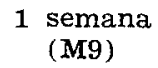 & $2 \underset{\text { semanas }}{(\mathrm{M} 10)}$ & $\begin{array}{c}1+2 \text { semanas } \\
(\text { M12) }\end{array}$ & \\
\hline & & & & \\
\hline M1: (2) & $0,733 *$ & $0,482^{* * *}$ & $0,709 *$ & $0,641 \div$ \\
\hline M2 & $0,727 *$ & $0,543 *$ & $0,738^{* *}$ & $0,699 \%$ \\
\hline M3 & 0,748 & $0,638 *$ & $0,799 \approx$ & 0,677 \\
\hline M4 & 0,086 & 0,074 & 0,090 & 0,073 \\
\hline M5 & 0,093 & 0,053 & 0,085 & 0,037 \\
\hline M6 & 0,667 & $0,431 *$ & $0,640 \approx$ & $0,560^{*}$ \\
\hline M7 & $0,635^{* *}$ & 0,481 & $0,646 *$ & 0,610 \\
\hline M8 & $0,722^{* *}$ & $0,450 \div$ & 0,684 & 0,596 \\
\hline M13 & 0,604 & $0,441 \div$ & $0,607 *$ & $0,575 * *$ \\
\hline M14 & $0,593 *$ & $0,532^{* *}$ & $0,646 *$ & $0,633 *$ \\
\hline M15 (3) & 0,245 & 0,100 & 0,200 & 0,144 \\
\hline
\end{tabular}

(1) Ver abreviação dos métodos no quadro 1. (2) Conteído de matéria orgânica.

(3) Absorbância pela luz ultravioleta a $260 \mathrm{~nm}$.

** Significativo ao nivel de $1 \%$. 
QUADRO 6. Coeficientes de determinaçāo $\left(R^{2}\right)$ nas correlações entre nitrogênio determinado pelos vários métodos químicos e aquele mineralizado pela incubação aeróbica e anaeróbica e a relação $\mathrm{C}: \mathrm{N}$ (Nitrogênio Walkley-Black)

\begin{tabular}{|c|c|c|c|c|}
\hline \multirow{3}{*}{ Métodos (1) } & \multicolumn{4}{|c|}{ M17 } \\
\hline & \multicolumn{3}{|c|}{ Aeróbica } & \multirow{2}{*}{ 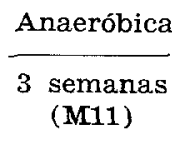 } \\
\hline & 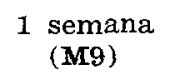 & $\begin{array}{c}2 \text { semanas } \\
\text { (M10) }\end{array}$ & $\begin{array}{c}1+2 \text { semanas } \\
(\mathrm{M} 12)\end{array}$ & \\
\hline & & $-\ldots$ & & - - - \\
\hline M1 (2) & $0,629 *$ & $0,429 * *$ & $0,616^{* *}$ & $0,540 * *$ \\
\hline M2 & $0,728^{* *}$ & $0,548 *$ & $0,740^{* *}$ & $0,703 *$ \\
\hline M3 & $0,749 \%$ & $0,643^{* *}$ & $0,801 *$ & $0,681 * *$ \\
\hline M4 & 0,081 & 0,066 & 0,083 & 0,070 \\
\hline M5 & 0,076 & 0,033 & 0,063 & 0,025 \\
\hline M6 & $0,666^{* *}$ & $0,431 *$ & $0,639 *$ & $0,557 * *$ \\
\hline M7 & $0,638 *$ & $0,489 \div *$ & 0,651 & $0,624^{* *}$ \\
\hline M8 & $0,717 *$ & $0,449 *$ & $0,680^{*} \div$ & $0,590 * *$ \\
\hline M13 & $0,622 * *$ & $0,443 * *$ & $0,620 *$ & $0,562 * *$ \\
\hline M14 & $0,586 *$ & $0,523 * *$ & $0,636^{*}$ & $0,612 * *$ \\
\hline M15 (3) & 0,239 & 0,090 & 0,191 & 0,142 \\
\hline
\end{tabular}

(1) Ver abreviação dos métodos no quađro 1. (2) Conteúdo de matéria orgânica. (3) Absorbância pela luz ultravioleta a $260 \mathrm{~nm}$.

** Significativo ao nível de $1 \%$.

Mesmo que não haja procedimento estatístico para determinar se os valores de $\mathrm{R}^{2}$ são diferentes, eles podem ser avaliados com base no grau de consistência e correlação. Dos métodos químicos, hidrólise com $\mathrm{Ca}(\mathrm{OH})_{2}$ (M3) e nitrogênio total (M2) deram os mais altos valores de $\mathrm{R}^{2}$, seguindo-se, próximo, a matéria orgânica (M1) e, depois, a digestão com permanganato alcalino (M14), nitrogênio pelo Walkley-Black (M13) e os procedimentos de autoclavagem (M6, M7, M8). Em último, vêm o nitrogênio inorgânico inicial (M4), amônio trocável (M5) e absorção pela luz ultravioleta (M15).

No entanto, quando se correlacionaram as quantidades de nitrogênio determinadas pelos métodos biológicos com as de matéria orgânica mais a relação $\mathrm{C}: \mathrm{N}$, obteve-se uma equação de regressão múltipla (não apresentada neste trabalho), sempre do tipo:

$$
\mathrm{Y}=\mathrm{b}_{0}+\mathrm{b}_{1} \mathrm{X}_{1}-\mathrm{b}_{2} \mathrm{X}_{2}
$$

onde:

$\mathrm{Y}$ : representa as quantidades de nitrogênio determinadas pelos métodos biológicos;

$X_{1}$ : representa o teor de matéria orgânica no solo;

$\mathrm{X}_{2}$ : representa a relação $\mathrm{C}: \mathrm{N}$ do solo. 
A equação de regressão múltipla, do tipo acima obtida, é indicativa de que, quanto maior for a relação $\mathrm{C}: \mathrm{N}$, para um mesmo teor de matéria orgânica no solo, menor será a quantidade de nitrogênio supostamente disponivel no solo às plantas. Por outro lado, de acordo com BREMNER (4) e RUSSEL (15), é perfeitamente conhecido que a velocidade e extensāo da mineralização do nitrogênio orgânico do solo, pelo menos em períodos curtos e intermediários, pode ser grandemente afetada pela relação C:N. Essa é uma razão por que os métodos biológicos, inerentemente, são superiores aos químicos na predição da disponibilidade do nitrogênio orgânico do solo.

\section{CONCLUSÕES}

O presente estudo permitiu concluir que qualquer dos seguintes métodos químicos pode ser satisfatoriamente usado para predizer a disponibilidade do nitrogênio orgânico do solo, em análisse de solo de rotina no Estado do Mississipi: matéria orgânica mais relação $C: N$, nitrogênio pelo Walkley-Black, hidrólise com $\mathrm{Ca}(\mathrm{OH})$.2 e digestāo corn permanganato alcalino. Recursos em instrumentos poderão constituir fator principal na seleção de um dado método químico. Entretanto, se um eletrodo de amônia puder ser adaptado nas determinaçōes de rotina de nitrogênio pelo método Walkley-Black, o uso da matéria orgânica mais a relação $C: N$ poderá ser preferido, porque as determinações de matéria orgânica estão-se tornando prática de rotina nos laboratórios de solos no Mississipi, como em outros Estados.

A autoclavagem dos solos em $\mathrm{CaCl}_{2}$ 0,01M por 16 horas a $121^{\circ} \mathrm{C}$ não se mostrou como o mais efetivo método químico para predizer a disponibilidade do nitrogênio orgânico naqueles solos, contrariamente aos resultados das determinações de SMITH \& STANFORD (17).

\section{SUMMARY}

\section{EVALUATION OF SOME CHEMICAL METHODS FOR PREDICTING THE AVAILABILITY OF ORGANIC SOIL NITROGEN}

A study was conducted to evaluate selected chemical methods for assessing the availability of organic soil nitrogen in Mississippi soils using nitrogen mineralized by aerobic and anaerobic incubation under controlled conditions as standards for comparison. Sixty-two soil samples were collected from agricultural and non-agricultural sites in representative physiographic areas of Mississippi. The soils varied greatly in texture, $\mathrm{pH}$, management practices, and organic matter content. The chemical methods evaluated were: organic matter (M1); total nitrogen (M2); $\mathrm{Ca}(\mathrm{OH})_{2}$ hydrolysis (M3); initial inorganic nitrogen (M4); exchangeable ammonium (M5), autoclaving and washing with $1 \mathrm{M} \mathrm{KCl}$ (M6); autoclaving and washing with $0.01 \mathrm{M} \mathrm{CaCl}_{2}$ (M7); autoclaving in $0.01 \mathrm{M} \mathrm{CaCl}_{2}$ and washing with $1 \mathrm{M} \mathrm{KCl}$ (M8); Walkley-Black nitrogen (M13); alkaline permanganate digestion (M14); and ultraviolet absorption (M15). The biological methods, used as standards for evaluation 
of the chemical methods, were: aerobic mineralization - 1 week (M9); aerobic mineralization - 2 weeks (M10); aerobic mineralization -1 week plus 2 weeks (M12) ; and anaerobic mineralization 3 weeks (M11). Except for initial inorganic nitrogen (M4), exchangeable ammonium (M5), and ultraviolet c bsorption by NaHCO, soil extracts (M15), all the chemical methods were moderately to highly correlated with each other, and, therefore, may be divided into two groups: the autoclaving methods (M6, M7, M8) and the other methods (M1, M2, M3, M13, M14). The highest correlation obtained with the chemical methods was between total nitrogen (M2) and $\mathrm{Ca}(\mathrm{OH})_{2}$, hydrolysis (M3). Of the chemical methods only initial inorganic nitrogen (M4) and exchangeable ammonium (M5) were not highly statistically correlated with the biological methods. The chemical methods were correlated to at lower degree with aerobic mineralization by M10 than with other bislogical methods. Based on degree and consistency of correlation, the chemical methods were ranked numerically as to their effectiveness in the following order, from simple correlation analysis: $\mathrm{Ca}(\mathrm{OH})$., hydrolysis $(\mathrm{M} 3)>$ total nitrogen $(\mathrm{M} 2)>$ alkaline permanganate digestion (M14) > Walkley-Black nitrogen (M13) > autoclaving procedures (M6, M7, M8) > organic matter (M1) > ultraviolet absorption by NallCO. soil extracts (M15). When the $\mathrm{C}: \mathrm{N}$ ratio was included with the biological methods in a multiple regression analysis, only the effectiveness of organic matter was increased substantially. Consequently, the order of effectiveness as established by simple correlation analysis was unchanged except that of organic matter (M1) that was brought among the chemical methods having the highest correlation with the biological methods.

\section{REFERENCIAS BIBLIOGRAFTCAS}

1. BARYOSEF, B. \& AKIRI, B. Sodium bicarbonate extraction to estimate nitrogen, phosphorus, and potassium availability in soils. Soil Science Society of America Journal, 42:319-323, 1978.

2. BREMNER, J. M. Determination of nitrogen in soil by the Kjeldahl method. Journal Agricultural Science, 55:11-33, 1960.

3. Nitrogen availability indexes. In: BLACK, C. A. et alii, eds. Methods of Soil Analysis. Madison, American Society of Agronomy, 1965. p.1324-1345. (Agronomy, Series of monographs, 9)

4. CORNFIELD, A. H. Ammonia released on treating soils with 1N sodium hydroxide as a possible means to predicting the nitrogen-supplying power of soils. Nature, 187:260-261, 1960.

5. FITTS, J. W.; BARTHOLOMEW, W. V.; HEIDEL, H. Correlation between nitrifiable nitrogen and yield response of corn to nitrogen fertilization on Iowa soils. Soil Science Society of America Proceedings, 17:119-122, 1953.

6. FOX, R. H. \& PIEKIELEK, W. P. Field testing of several nitrogen availability indexes. Soil Science Society of America Journal, 42:474-750, $1978 \mathrm{a}$.

7. _ \& A rapid method for estimating the nitrogen-supplying culpability of a soil. Soil Science Society of America Journal, 42:751-753, 1978b.

8. KEENEY, D. R. \& BREMNER, J. M. A chemical index of soil nitrogen availability. Nature, 211:892-893, 1966.

9. LANCASTER, J. D. Rapid determination of ammonium in soils. Mississippi Agricultural and Forestry Experiment Station MAFES, 1973. 5p. (Mimeo material)

10. MacLEAN, A. A. Measurement of soil nitrogen-supplying fower of soils by extraction with sodium bicarbonate. Nature, 203:1307-1308, 1964. 
11. MUNSON, R. D. \& STANFORD, G. Predicting nitrogen fertilizer needs of Iowa soils: IV. Evaluation of nitrate production as a criterion of nitrogen availability. Soils Seience Society of America Proceedings, 19:446-468, 1955.

12. POWERS, R. F. Mineralizable soil nitrogen as an index of nitrogen availability to forest trees. Soil Science Society of America Journal, 44:1314-1320, 1980.

13. PRASAD, R. Development of a chemical method for determining potentially available nitrogen in soils. Mississippi, State University, Agronomy Department, 1961. 87p. Dissertation. (Ph.D.)

14. PROCEDURES used by the Auburn University Soil Testing Laboratory. Department of Agronomy and Soils, 1974. 17p. (Departmental Series, 16)

15. RUSSEL, E. W. Soil conditions and plant growth. 10ed. In: The decomposition of the soil organic matter. London, Longman, 1973. cap. 15 , p. 282.

16. SAHRAWAT, K. L. \& PONNAMPERUMA, F. N. Measurement of exchangeable ammonium in tropical rice soils. Soil Science Society of America Journal, 42:282-283, 1978.

17. SMITH, S. J. \& STANFORD, G. Evaluation of a chemical index of soil nitrogen availability. Soil Science, 111:228-232, 1971.

18. — ; YUNG, L. B.; MILLAR, G. E. Evaluation of soil nitrogen mineralization potentials under modified field conditions. Soil Science Society of America Journal, 41:74-76, 1977.

19. STANFORD, G. Extraction of soil organic nitrogen by autoclaving in water: 2. A kinetic approach to estimating the $\mathrm{NaOH}$ - distillable fraction. Soil Science, $107: 323-338,1969$.

20. —— CARTER, J. N.; SMITH, S. J. Estimates of potentially mineralizable soil nitrogen based on short-term incubations. Soil Science Society of America Proceedings, 38:99-102, 1974.

21. —— DEMAR, W. H. Extraction of soil organic nitrogen by autoclaving in water: I. The $\mathrm{NaOH}$ - distillable fraction as an index of nitrogen availability in soils. Soil Science, 107:203-205, 1969.

22. —_ \& HANWAY, J. Predicting nitrogen fertilizer needs of Iowa soils: II. A simplified technique for determining relative nitrate production in soils. Soil Science Society of America Proceedings, 19:74-77, 1955.

23. —_ \& SMTTH, S. J. Estimating potentially mineralizable soil nitrogen from a chemical index of soil nitrogen availability. Soil Science, 122:71-76, 1976 . 